\title{
Comparative Transcriptome Analysis of Isoetes Sinensis Under Terrestrial and Submerged Conditions
}

\author{
Tao Yang ${ }^{1} \cdot$ Xing Liu ${ }^{1}$
}

Published online: 27 June 2015

(C) The Author(s) 2015. This article is published with open access at Springerlink.com

\begin{abstract}
Isoetes L. is an ancient genus of heterosporous lycopsids with a unique phylogenetic position. Repeated adaptations to environmental changes over time have contributed to occupying a variety of niches in Isoetes. However, we know little about how they adapt to the environmental changes, and the sequence resources are very limited in public databases. Isoetes sinensis is an amphibious plant in this genus, alternating frequently between terrestrial and aquatic environments. In this study, I. sinensis was applied to investigate the adaptations under terrestrial (TC) and submerged (ST) conditions using Illumina RNA-sequencing technology. Approximately 87 million high-quality reads were yielded and assembled into 31,619 unigenes with an average length of $1618 \mathrm{bp}$. Overall, 28,208 unigenes were annotated against the National Center of Biotechnology Information (NCBI), Non-redundant $(\mathrm{Nr})$, Cluster of Orthologous Groups (COG), Gene Ontology (GO), and Kyoto Encyclopedia of Genes and Genomes (KEGG) databases. Moreover, we identified 1740 differentially expressed genes with 1146 up-regulated and 594 downregulated genes under TC. GO annotation revealed that stress-relevant categories were remarkably enriched, and KEGG enrichment analysis showed that the phytohormone signalings and carbohydrate metabolism were significantly influenced. Furthermore, a total of 1646 transcription factors (TF) were identified and classified into $54 \mathrm{TF}$ families; among
\end{abstract}

Electronic supplementary material The online version of this article (doi:10.1007/s11105-015-0906-6) contains supplementary material, which is available to authorized users.

Xing Liu

xingliu@whu.edu.cn

1 Laboratory of Plant Systematics and Evolutionary Biology, College of Life Science, Wuhan University, Wuhan, Hubei 430072, China them, 180 TFs were dynamic between terrestrial and submerged conditions. This study is the first report for Isoetes to generate numerous sequences and establish general understandings about the adaptations in the changing environments. The dataset provides a foundation for novel gene discoveries, comparative genomics, functional genomics, and phylogenetics in Isoetes.

Keywords Isoetes L. I Isoetes sinensis · Transcriptome · Submerged condition $\cdot$ Terrestrial condition $\cdot$ Abiotic stress

\section{Background}

Isoetes L. (family Isoetaceae, quillworts) is an ancient genus of heterosporous lycopsids with a unique position in plant evolution (Pigg 1992). Phylogenetic analyses show that Isoetes is one of the earliest basal vascular plants, which can date back to the Devonian Period (Foster and Gifford 1959; Pigg 2001). Isoetes has approximately 200 species characterized by a strong reduced plant body (Li et al. 2012; Pigg 1992; Pigg 2001). Furthermore, this genus is the only survival of ancient taxa as the closest relatives of the famous tree lycopods (Hoot and Taylor 2001; Pigg 1992; Pigg 2001). Since the Paleozoic Era, repeated adaptations to environmental changes over time have contributed Isoetes to range from evergreen aquatics to ephemeral terrestrials. To date, the genus occupies a variety of niches, including oligotrophic soft-water lakes, higher altitude wetlands, seasonal pools, and intermittent streams (Keeley 1987; Pigg 1992; Pigg 2001; Taylor and Hickey 1992). The habitat preferences reflect past adaptations to the environmental changes, and play an essential role in the phylogenetic evolution of Isoetes (Keeley 1987; Pigg 1992; Taylor and Hickey 1992). Nonetheless, we know little about how Isoetes adapt to the environmental changes. Moreover, 
sequence resources are very limited in the National Center of Biotechnology Information (NCBI) database.

Isoetes sinensis Palmer is an allotetraploid plant $(2 n=4 x=$ 44) distributed in East Asia (Liu et al. 2001). Recent phylogenetic research has indicated that $I$. sinensis probably derives from hybridization between the diploid I. yunguiensis $(2 n=$ $22)$ and $I$. taiwanensis $(2 n=22)$ (Liu et al. 2004). In addition, I. sinensis is a typical amphibious plant, which grows mainly in seasonal pools or intermittent streams and alternates frequently between terrestrial and aquatic environments (Keeley 1987; Wang et al. 2006). Thus, I. sinensis is a good model for Isoetes to research the adaptations to the environmental changes.

High-throughput sequencing technology makes it possible for non-model plants to produce multiple sequences at a relatively low cost. De novo assembly has been proven to be an ideal method for short reads in non-model organisms (Haas et al. 2013). In addition, RNA-sequencing approach has become a popular method to discover novel genes and explored different expression profiles under various conditions. In the last few years, there were substantial reports in RNAsequencing datasets and expression profiles from lower to higher species (Der et al. 2011; Shih et al. 2014). In this study, our main objectives are to obtain numerous sequence resources and establish general understandings about how the Isoetes species adapt to the environmental changes. cDNA libraries from juvenile leaves of $I$. sinensis under terrestrial (TC) and submerged (SC) conditions were sequenced on an Illumina Hiseq 2000 platform, respectively. The dataset yielded multiple sequence resources and plotted a dynamic expression profile under TC and SC. GO annotation revealed that stress-relevant categories were remarkably enriched, and KEGG enrichment analysis showed that the phytohormone signalings and carbohydrate metabolism were significantly influenced. We further analyzed transcription factors (TF) to reveal their potential functions in I. sinensis for adapting to the environmental changes.

\section{Materials and Methods}

\section{Plant Materials and Growth Conditions}

I. sinensis was collected from Xinan River with a fluctuated water level in Zhejiang Province, China (29 $28^{\prime} \mathrm{N} ; 119^{\circ} 14^{\prime}$ E). The plants were cultivated in the greenhouse of Wuhan University. All materials initially bred under SC with approximately $60 \mathrm{ml}$ tap water for a month. Some experimental materials still maintained under SC about $4 \mathrm{~cm}$ below the surface of "artificial floodwater", which were defined as control groups in this study. The others were transferred to TC for an additional month, and all leaves were entirely exposed to the air. Owing to the important roles of plant leaves in adapting to the changing environments (Baerenfaller et al. 2012), juvenile leaves under TC and SC were immediately harvested, frozen in liquid nitrogen, and stored at $-80{ }^{\circ} \mathrm{C}$, respectively.

\section{RNA Isolation and Sequencing}

Total RNA was isolated using Trizol (Invitrogen Inc., USA), and the residual DNA was removed using RNase-free DNase I (Takara, Da Lian, China) according to the manufactures' protocols. The RNA quantity was checked using ND-1000 Nanodrop Spectrophotometer (Thermo Scientific, DE, USA), and the quality was verified using 2100 Bioanalyzer RNA Nanochip (Aligene, CA, USA). Ten micrograms of verified total RNA was pooled in an equal amount from three independent extractions into a combined sample. The DNAfree mRNA was captured by magnetic oligo(dT) beads, fragmented to a size of $200 \mathrm{bp}$, and then synthesized into the first strand cDNA with random hexamer primers. The second strands were further synthesized using RNase H (New England Biolabs Inc., Ipswich, MA, USA) and DNA polymerase (Invitrogen, Carlsbad, CA, USA). We further repaired the end fragments and ligated them with sequencing adaptors. Suitable fragment ranges for PCR application $(200 \pm 25 \mathrm{bp})$ were selected by agarose gel electrophoresis and purified using a QIAquick PCR extraction kit (Qiagen, USA). The constructed cDNA libraries were sequenced on an Illumina Hiseq 2000 platform following the manufacturers' instructions (Illumina Inc., San Diego, CA, USA).

\section{De Novo Assembly and Functional Analysis of Transcriptome Sequencing Results}

The raw reads initially performed a stringent quality control analysis using FastQC (Andrews 2010). The base quality thresholds contained removing adaptors, sequence-specific bias, polymerase chain reaction artifacts, ambiguous nucleotide reads with $\mathrm{N}$, and low quality bases with average Phred scores less than 20. After filtering and trimming, all clean reads were merged and further de novo assembled into unigenes using Trinity program, setting k-mers length to 25 (Grabherr et al. 2011). Then all unigenes were aligned using BLASTx against the non-redundant $(\mathrm{Nr})$ database with an $E$ value cutoff of $\mathrm{e}^{-05}$. To annotate the unigenes which failed to be aligned to the $\mathrm{Nr}$ database, we further applied GetORF software to predict their orientations and underlying protein coding regions (Rice et al. 2000). All assembled sequences were further searched using BLASTx against the Gene Ontology (GO), Cluster of Orthologous Groups (COG), and Kyoto Encyclopedia of Genes and Genomes (KEGG) databases with an $E$ value cutoff of $\mathrm{e}^{-05}$. Blast2GO was used to obtain GO annotations, and WEGO database was applied to give a broad overview of the annotations regarding biological process 
(BP), molecular function (MF), and cellular component (CC) (Ye et al. 2006). COG database was used to represent a phylogenetic lineage of the I. sinensis transcriptome. Moreover, KEGG database was employed to explore putative pathways using KEGG automatic annotation service (KAAS) with the bi-directional best hit method (Kanehisa 2002).

\section{Identification and Functional Characterization of Differentially Expressed Genes}

To investigate dynamic expression profiles under TC and SC, the present frequency of each unigene was calculated and normalized into reads per kilobase per million mapped reads (RPKM) values (Mortazavi et al. 2008). The expression fold changes were calculated as the $\log _{2}$ ratio of the two RPKM values under TC and SC. False positive and negative errors were analyzed by calculating the false discovery rate (FDR) values, which also applied to adjust $p$ values using $\mathrm{R}$ program. FDR significant scores $\leq 0.001$ and $\left|\log _{2}\left(\mathrm{RPKM}_{\mathrm{TC} / \mathrm{SC}}\right)\right| \geq 1$ were used as the thresholds to identify DEGs. In addition, all DEGs were further used for GO annotations and KEGG enrichment analysis. GO Slim was performed to map the GO annotations to a plant GO Slim file using the Fisher's tests with the cutoffs as FDR adjusted $p$ values less than 0.01 . We also used a hypergeometric test to assess significant enrichment levels by setting $p$ values less than 0.05 .

\section{Identification and Analysis of TFs}

To identify putative TFs in the transcriptome, we downloaded all well-known TF protein sequences from the Plant Transcriptional Factor (PlnTFDB) Database (Riaño-Pachón et al. 2007). All unigenes and DEGs were subjected to a local BLASTx homology search against the well-known TFs, respectively. The threshold of default parameters is an $E$ value cutoff of $\mathrm{e}^{-10}$. The top hits were further extracted from the BLASTx results using an in-house Perl script, which were considered as the putative TFs in this dataset.

\section{Validation of the Transcriptomic Results Using Quantitative Real-Time PCR}

To validate the reliability of the transcriptomic results, 27 DEGs were selected for qRT-PCR tests (Bustin et al. 2009). Total RNA from the juvenile leaves under TC and SC was extracted using RNAiso Plus (Takara, Japan) according to the manufacturers' instructions. Total RNA of $1.5 \mu \mathrm{g}$ was reversetranscribed into single strand cDNA using Primerscript ${ }^{\mathrm{TM}}$ One Step RT-PCR Kit Ver. 2 (Takara, Japan). Gene-specific primers were designed using a free online primer design tool (http://primer3plus.com/cgi-bin/dev/primer3plus.cgi). The qRT-PCR analysis was performed on a CFX96 real-time PCR system (Bio-Rad, Hercules, USA). The cDNA was diluted tenfold and amplified in a $25-\mu \mathrm{l}$ solution, containing $12.5 \mu 12 \times$ SYBR premix, 0.25 pmol forward and reverse primers, $2.5 \mu \mathrm{l}$ diluted cDNA, and $7.0 \mu \mathrm{l}$ sterile water. The PCR program was $95^{\circ} \mathrm{C}$ for 3 min for the initial denaturation, followed by 40 cycles of $95^{\circ} \mathrm{C}$ for $30 \mathrm{~s}$, annealing temperatures for $15 \mathrm{~s}$, and finally $72{ }^{\circ} \mathrm{C}$ for $30 \mathrm{~s}$. Annealing temperatures and related information about the DEGs are listed in Table S1. Melting curves were plotted to determine the specificity at the end of the PCR cycling over the range $65-95^{\circ} \mathrm{C}$. Baseline and threshold cycle $(\mathrm{Ct})$ were determined automatically by Bio-Rad CFX Manager 2.1 software. The relative expression levels were calculated using $2^{-\Delta \Delta \mathrm{Ct}}$ method (Livak and Schmittgen 2001) and normalized to the geometric average of $\mathrm{Ct}$ values with Actin as an internal control gene. All experiments and analyses were conducted in triplicate.

\section{Results}

\section{Sequencing and De Novo Assembly}

To develop a general view of the $I$. sinensis transcriptome, cDNA libraries from the juvenile leaves under TC and SC were sequenced on an Illumina Hiseq 2000 platform, respectively. In total, we obtained 47,905,510 raw pair-end reads under TC and 52,709,149 under SC, respectively (Table S2). After filtering and trimming, approximately 87 million clean reads were generated, corresponding to $41,344,520$ clean reads under TC and 45,530,550 under SC, respectively. All clean reads are deposited in the NCBI Short Read Archive database. The accession numbers are SRR1646513 under TC and SRR1648119 under SC. Then all clean reads were merged and de novo assembled into 31,619 unigenes with a mean length of $1618 \mathrm{bp}$ and half of the assembled length (N50) of 2350 bp (Table S3). In addition, the size distribution of unigenes ranged from 200 to more than $5000 \mathrm{bp}$ (Fig S1). A total of $78.3 \%$ unigenes were larger than $500 \mathrm{bp}$, and $31.1 \%$ were larger than $2000 \mathrm{bp}$.

\section{Annotation and Functional Characterization of I. Sinensis Transcriptome}

All assembled unigenes were further searched using BLASTx against the Nr, GO, COG, and KEGG databases (Table 1). A total of $28,208(89.2 \%)$ unigenes were similar to the known sequences in the $\mathrm{Nr}$ database with an $E$ value cutoff of $\mathrm{e}^{-05}$ (Fig. 1). Overall, $15 \%$ unigenes had strong similarities $(E$ value $<10 \mathrm{e}^{-100}$ ) and $65 \%$ presented moderate similarities $\left(10 \mathrm{e}^{-100}<E\right.$ value $\left.<10 \mathrm{e}^{-10}\right)$. In addition, 23,020 unigenes were assigned to at least one GO annotations using Blast2GO program. WEGO database was further used to map the annotations to particularly functional classification in terms of BP, $\mathrm{MF}$, and CC. GO annotations of 52,492 were within $23 \mathrm{BP}$ 
Table 1 Functional annotations of unigenes from BLASTx searches against the $\mathrm{Nr}, \mathrm{GO}$, COG, KEGG, and PlnTFDB databases

\begin{tabular}{lll}
\hline Databases & Numbers & Percentages \\
\hline $\mathrm{Nr}$ & 28,208 & 89.2 \\
GO & 23,020 & 72.8 \\
COG & 26,388 & 83.5 \\
KEGG & 7964 & 28.2 \\
PlnTFDB & 1646 & 5.8 \\
\hline
\end{tabular}

terms, 31,141 were within $14 \mathrm{MF}$ terms, and 62,066 were within $16 \mathrm{CC}$ terms (Fig S2). The most abundant term in BP was "metabolic process", followed by "cellular process", "response to stimulus", and "biological regulation". A large number of unigenes in MF were related to "binding" and "catalytic". The commonest terms in CC were "cell" and "cell part".

All unigenes were further used to phylogenetically describe functional features using the COG database. A total of 26,388 unigenes were assigned to $25 \mathrm{COG}$ classifications (Fig. 2). Ignoring "function unknown" category, the largest group was "general function prediction only" (18\%), followed by "posttranslational modification, protein turnover, chaperones" (7 \%), and "signal transduction mechanisms" (6\%). The smallest groups were "nuclear structure" $(0.18 \%)$, "extracellular structures" $(0.13 \%)$, and "cell motility" $(0.05 \%)$.

To further elucidate the biological processes, the annotated unigenes were mapped into the reference pathways against the KEGG database. Unigenes of 7964 were annotated into 126 biological pathways in the KEGG database (Table S4).
Unigenes related to "metabolic pathways" (1743 unigenes) were the largest, followed by "biosynthesis of secondary metabolites" (913 unigenes), "ribosome" (341 unigenes), and "plant hormone signal transduction" (255 unigenes).

\section{Identification and Functional Analysis of DEGs}

We determined 1740 DEGs with the thresholds of FDR value $\leq 0.001$ and $\left|\log _{2}\left(\mathrm{RPKM}_{\mathrm{TC} / \mathrm{SC}}\right)\right| \geq 1$. Comparing with $\mathrm{SC}$, a total of 1146 DEGs were up-expressed and 594 were downexpressed under TC. To systematically characterize I. sinensis in response to the environmental changes, all DEGs were further used to investigate the GO annotations and KEGG enrichment pathways. We also applied a plant GO Slim file to give a broad overview of the GO annotations using Fisher's tests with the cutoffs as $p$ values less than 0.01 . Of the 1740 DEGs, 1374 were assigned to 9184 GO annotations using Blast2GO. Moreover, 960 DEGs were significantly categorized to $4 \mathrm{MF}$ terms, 3160 were within $21 \mathrm{CC}$ terms, and 3035 were within 22 BP terms (Fig. 3). Large numbers of DEGs were significantly enriched in functional terms, such as "sequence-specific DNA binding transcription factor activity", "response to abiotic stimulus", "response to endogenous stimulus", "response to stress", "extracellular regions", "vacuole", and "membrane".

We used a hypergeometric test to assign the DEGs to terms in the KEGG database. Of the 1740 DEGs, 386 were assigned and grouped into 96 KEGG pathways (Table S5). A total of $76.94 \%$ DEGs were related to "metabolism" category, followed by "environmental information processing" (15.80\%), and "cellular processes" (9.85\%) (Fig. 4).
Fig. $1 E$ value distribution of unigenes based on a BLASTx search against the $\mathrm{Nr}$ database

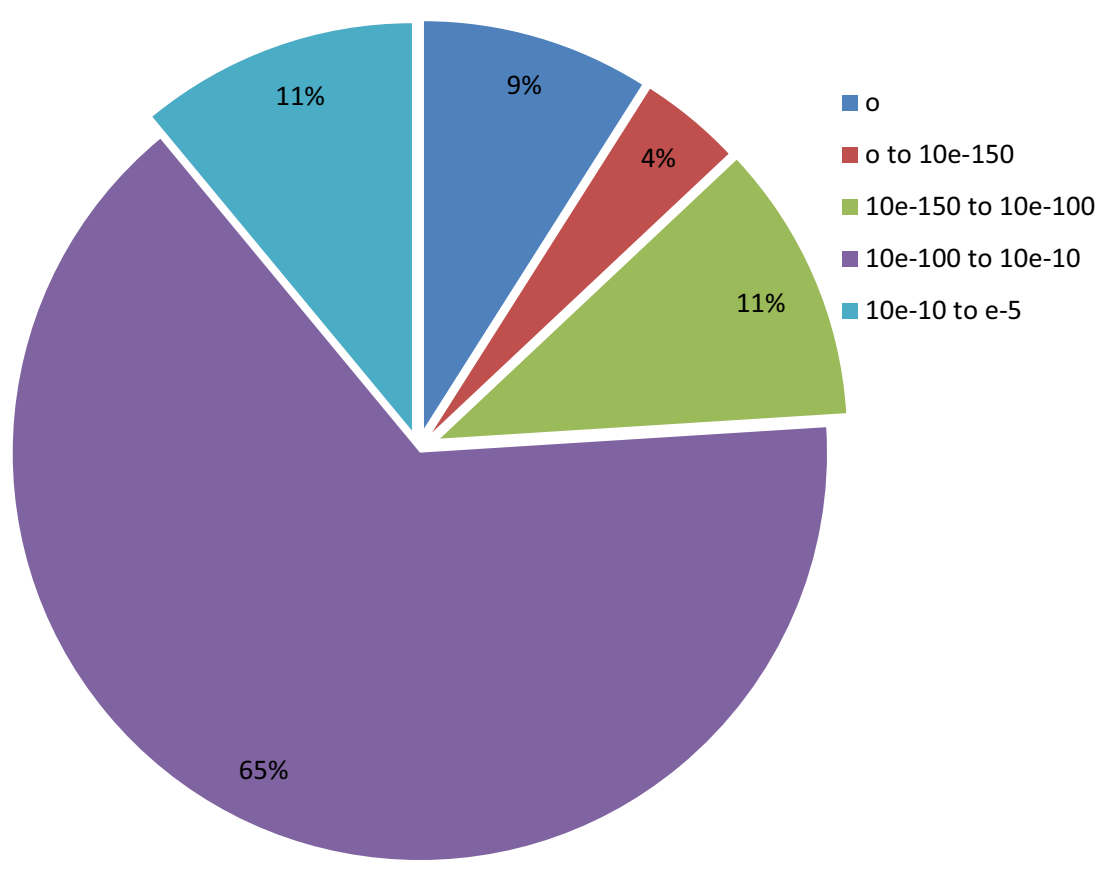


Fig. 2 COG categories of assembled unigenes

Fig. 3 GO Slim functional classifications of DEGs. DEGs associate with $\mathrm{GO}$ annotations are represented regarding biological process $(B P)$, cellular component $(C C)$, and molecular function $(M F) . X$ axis indicates the functional significance of the enriched terms. Y axis describes the related GO terms. The width of the lines is the level of enriched numbers in each GO term, suggesting that the wider lines share more DEGs numbers in each term

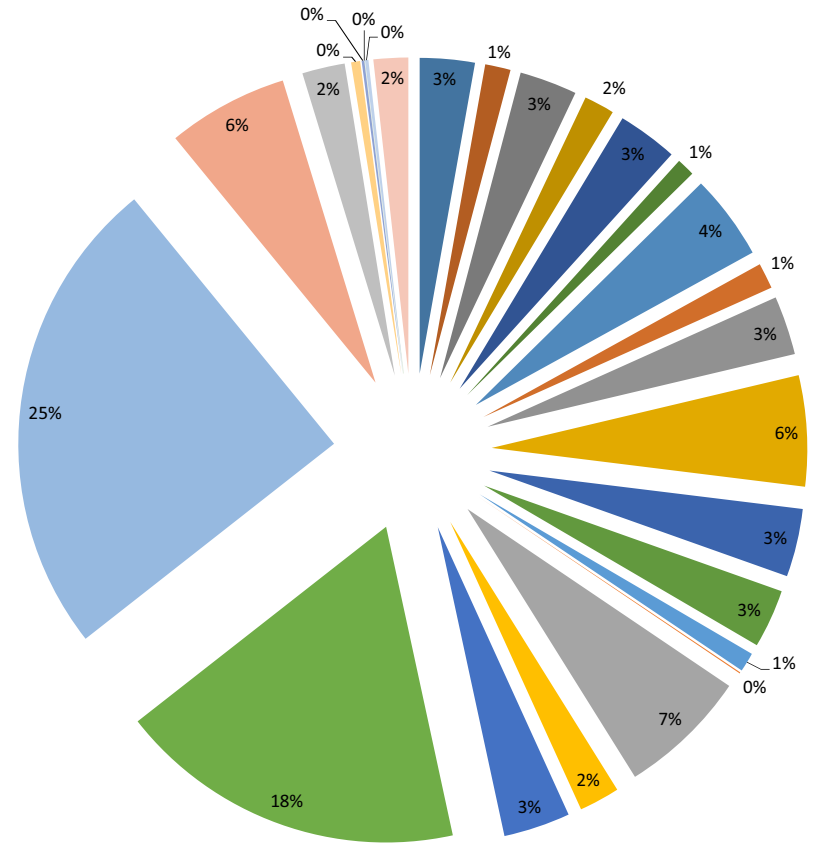

- RNA processing and modification

- Chromatin structure and dynamics

- Energy production and conversion

- Cell cycle control, cell division, chromosome partitioning

- Amino acid transport and metabolism

- Nucleotide transport and metabolism

- Carbohydrate transport and metabolism

- Coenzyme transport and metabolism

- Lipid transport and metabolism

- Translation, ribosomal structure and biogenesis

- Transcription

- Replication, recombination and repair

- Cell wall/membrane/envelope biogenesis

n Cell motility

- Posttranslational modification, protein turnover, chaperones

- Inorganic ion transport and metabolism

- Secondary metabolites biosynthesis, transport and catabolism

- General function prediction only

- Function unknown

- Signal transduction mechanisms

- Intracellular trafficking, secretion, and vesicular transport

Defense mechanisms

Extracellular structures

- Undetermined

Nuclear structure

- Cytoskeleton sequence-specific DNA binding transcription factor activity -
response to abiotic stimulus response to endogenous stimulustransportbehavior -

response to external stimulus biological_process-

response to stress
response to biotic stimulus -

response to biotic stimulus -

post-embryonic development multicellular organismal development -

metabolic prowth-
anatomical structure morphogenesis enzyme regulator activity -

enzyme reguly reproduction cellular process -

cellar pricess pollination -

extracellular region vacuole
val region

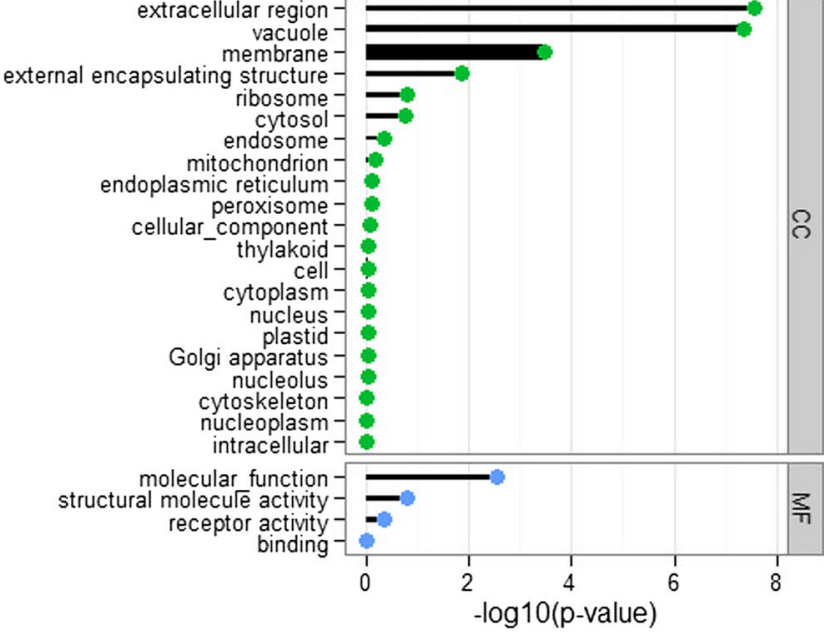




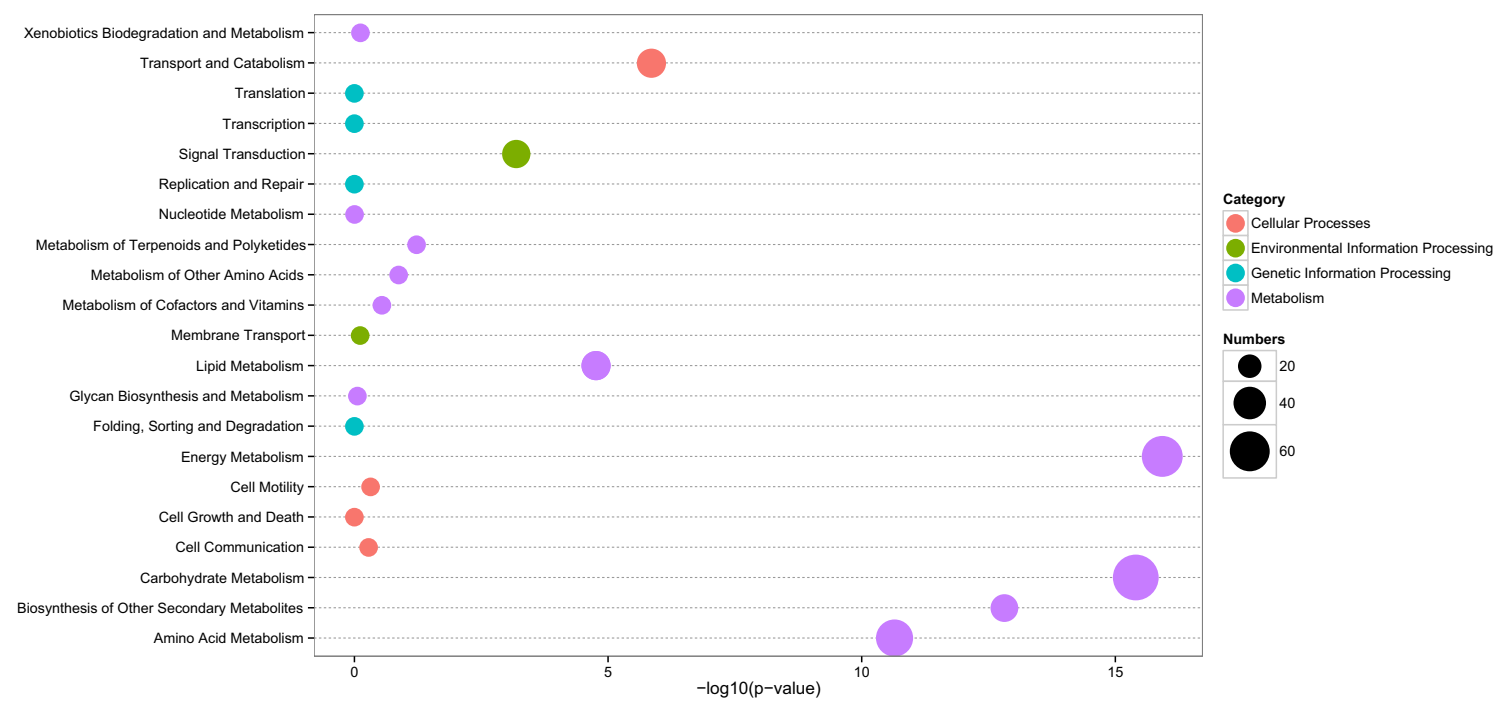

Fig. 4 KEGG pathway assignments of DEGs. KEGG pathways contain the cellular process (red points), environmental information processing (green points), genetic information processing (blue points), and metabolism (purple points) categories. $X$ axis indicates the functional significance of the enriched terms. Y axis is the KEGG pathway terms. The point sizes describe the enriched numbers in each term
Furthermore, the DEGs in the "metabolism" category was mainly over-represented in "carbohydrate metabolism" ( $p$ value $3.95 \mathrm{e}^{-16}$ ), followed by "energy metabolism" ( $p$ value $\left.1.19 \mathrm{e}^{-16}\right)$, and "biosynthesis of other secondary metabolites" ( $p$ value $1.54 \mathrm{e}^{-13}$ ). In addition, phytohormone signalings were also markedly enriched in "environmental information processing", including ABA, ethylene, jasmonic acid (JA), salicylic acid (SA), cytokinin, auxin, and gibberellin signaling pathways (Table 2).

\section{Identification and Analysis of TFs}

In this study, we identified 1646 putative TFs using a local BLASTx homology search against the PlnTFDB. The 1646 TFs were classified into 54 out of 58 TF families in this dataset (Table 1). The most abundant TF family was MYB-related proteins, followed by NAC, C2H2, bHLH, ERF, MYB, $\mathrm{C} 3 \mathrm{H}$, and WRKY protein families (Table 3). To describe the dynamic regulations for TFs under TC and SC, all DEGs were also aligned to identify dynamic TF families. A total of 180 TFs were observed with 134 up-regulated and 46 downregulated TFs. Furthermore, the most enriched TF families in the up-regulated DEGs were NAC and MYB-related proteins, followed by WRKY, bHLH, MYB, and ERF protein families; whereas the most abundant TF family in the downregulated DEGs was bHLH protein, followed by MYB, AP2, B3, ERF, and MYB-related proteins (Table 3).

\section{Validation of the Transcriptome Using qRT-PCR}

To validate the transcriptomic results, we performed qRT-PCR analysis for 27 DEGs under TC and SC. Among the selected
DEGs, 13 DEGs were randomly selected, and the others were related to phytohormone signaling pathways (Table S1). Actin was used as an internal control gene in this study. We used a Pearson correlation analysis to compare different expression levels between the transcriptome and qRT-PCR. There was a high correlation coefficient between the qRT-PCR and transcriptome results with Pearson $r=0.9285$ (Fig. 5), further confirming that the transcriptomic results were acceptable.

\section{Discussions}

RNA-sequencing approach is an effective alternative for nonmodel plants to obtain a large number of resources, and the technology is sensitive to detect dynamic ranges of the genes expression. In this study, we obtained a total of approximately 87 million high-quality reads using Illumina pair-end sequencing technology. This is the first report for Isoetes to produce substantial sequences using next-sequencing technology. The transcriptome provided reliable resources that more than $99 \%$ reads passed the QC analysis. A high correlation coefficient was conducted between the results of qRT-PCR and transcriptome $\left(R^{2}=0.9256\right)$. In addition, the unigenes quality was favorable with commendable average length (1618 bp) and N50 length (2350 bp). To the best of our knowledge, average length and N50 of the unigenes were longer than the previous studies via comparing our transcriptome with previous de novo assembly sequencing data (Der et al. 2011; Gao et al. 2014; Shih et al. 2014).

Plants are sessile organisms, which need to face with environmental changes during the growth process. It is necessary for plants' flexibly in response to different stresses in order to 
Table 2 Information about phytohormone signaling pathways under terrestrial and submerged conditions

\begin{tabular}{|c|c|c|c|c|c|c|}
\hline GeneID & Pathway & Annotation & RPKM (T) & RPKM (S) & $-\log _{2}$ fold change & $p$ value \\
\hline comp48729_c1_seq1 & $\mathrm{ABA}$ & $A B F$ & 15.09 & 8.21 & 0.85 & 0.1742 \\
\hline comp51265_c0_seq1 & & $A B F$ & 40.45 & 14.40 & 1.46 & 0.0124 \\
\hline comp47239_c0_seq1 & & $P Y L$ & 42.17 & 189.52 & -2.20 & 0.0002 \\
\hline comp59632_c1_seq8 & Ethylene & CTR1 & 2.37 & 9.06 & -1.96 & 0.0021 \\
\hline comp53115_c1_seq2 & & ETR & 13.58 & 6.40 & 1.06 & 0.1021 \\
\hline comp54747_c0_seq2 & & $E B F 1 / 2$ & 165.81 & 34.87 & 2.22 & 0.0002 \\
\hline comp50190_c0_seq2 & JA & COI-1 & 37.81 & 6.81 & 2.45 & 0.0001 \\
\hline comp46730_c0_seq1 & & $J A Z$ & 68.65 & 8.21 & 3.04 & 0.0000 \\
\hline comp48472_c0_seq2 & & $J A Z$ & 14.34 & 2.19 & 2.68 & 0.0007 \\
\hline comp56477_c2_seq8 & & $J A R 1$ & 2.52 & 0.11 & 4.51 & 0.0002 \\
\hline comp52220_c0_seq2 & SA & NPR1 & 2.84 & 0.53 & 2.40 & 0.0058 \\
\hline comp44025_c1_seq1 & & PR1 & 0.23 & 5.34 & -4.57 & 0.0001 \\
\hline comp61194_c0_seq1 & & $P R 1$ & 0.43 & 6.73 & -4.00 & 0.0008 \\
\hline comp55638_c0_seq1 & & $T G A$ & 10.72 & 3.66 & 1.52 & 0.0143 \\
\hline comp47186_c0_seq1 & Cytokinin & AHK2_3_4 & 9.46 & 67.91 & -2.87 & 0.0000 \\
\hline comp60573_c0_seq18 & & AHK2_3_5 & 16.51 & 59.51 & -1.88 & 0.0013 \\
\hline comp39440_c0_seq1 & & $A H P$ & 34.34 & 7.22 & 2.22 & 0.0005 \\
\hline comp49013_c0_seq1 & & $A-A R R$ & 50.94 & 12.54 & 1.99 & 0.0007 \\
\hline comp48142_c0_seq1 & Auxin & $I A A$ & 34.60 & 76.08 & -1.16 & 0.0429 \\
\hline comp54483_c1_seq1 & & $S A U R$ & 0.32 & 1.80 & -2.53 & 0.0460 \\
\hline comp51132_c1_seq1 & & $A U X 1$ & 28.64 & 46.13 & -0.72 & 0.2170 \\
\hline comp51568_c0_seq6 & Gibberellin & GID1 & 12.34 & 4.24 & 1.51 & 0.0191 \\
\hline
\end{tabular}

Phytohormone signaling pathways are present in the dataset, including ABA, ethylene, JA, SA, cytokinin, auxin, and gibberellin

survive or reproduce offspring (Wang et al. 2003; Zeng et al. 2014). To date, the researches on environmental adaptations are mainly focused on higher plants or commercial crops (Hiz

Table 3 Statistics of total and differentially expressed transcription factors related to the most abundant 12 transcription factor families

\begin{tabular}{llll}
\hline TF families & All unigenes & Up regulated & Down regulated \\
\hline MYB related & 107 & 10 & 2 \\
NAC & 78 & 10 & 1 \\
C2H2 & 73 & 7 & 1 \\
bHLH & 72 & 8 & 5 \\
ERF & 59 & 7 & 2 \\
C3H & 53 & 0 & 0 \\
MYB & 53 & 7 & 3 \\
WRKY & 51 & 8 & 0 \\
E2F/DP & 41 & 3 & 1 \\
FAR1 & 39 & 1 & 0 \\
AP2 & 21 & 0 & 3 \\
B3 & 41 & 1 & 2 \\
Totals & 1646 & 134 & 46 \\
\hline
\end{tabular}

In this dataset, 1646 transcription factors (TF) are identified, and a total of 180 TFs are dynamic expressions with 134 up-regulated TFs and 46 down-regulated TFs et al. 2014). GO annotation revealed that stress-relevant categories were remarkably enriched with the environmental changes. The analyses expand global understandings for adaptations to the environmental changes in basal plants, and indicate that stresses also play essential roles for Isoetes in the changing environments.

Hormones are important regulators for plants to adapt to environmental changes (Shan et al. 2013). Pathway enrichment analysis showed that phytohormone signalings were markedly enriched in "environmental information processing", including ABA, ethylene, JA, SA, cytokinin, auxin, and gibberellin. ABA and ethylene are considered to be the most important phytohormones in response to abiotic stress (Peleg and Blumwald 2011). ABA is a central regulator in response to the environmental changes by triggering many stress-related genes and further increasing the associated tolerance (Chinnusamy et al. 2008). ABA regulations in the water-limited environments show that cellular dehydration increases trigger downstream targets, such as TFs, signaling factors, and the others (Yamaguchi-Shinozaki and Shinozaki 2006). ABA responsive element binding factor $(A B F)$ is a key signaling factor belonging to the basic-region/leucine zipper TF family (Yoshida et al. 2010). In this dataset, two $A B F$ homologues (comp48729_c1_seq1 and comp51265_c0_seq1) were identified and uniformly up-regulated under TC, suggesting 
Fig. 5 Correlation coefficient of fold changes between the transcriptome and qRT-PCR. The transcriptional levels of 27 DEGs are detected by qRT-PCR with three biological replications and technical replications. Actin is used as an internal control gene. A correlation coefficient of fold changes between transcriptome and qRT-PCR is calculated by R programs

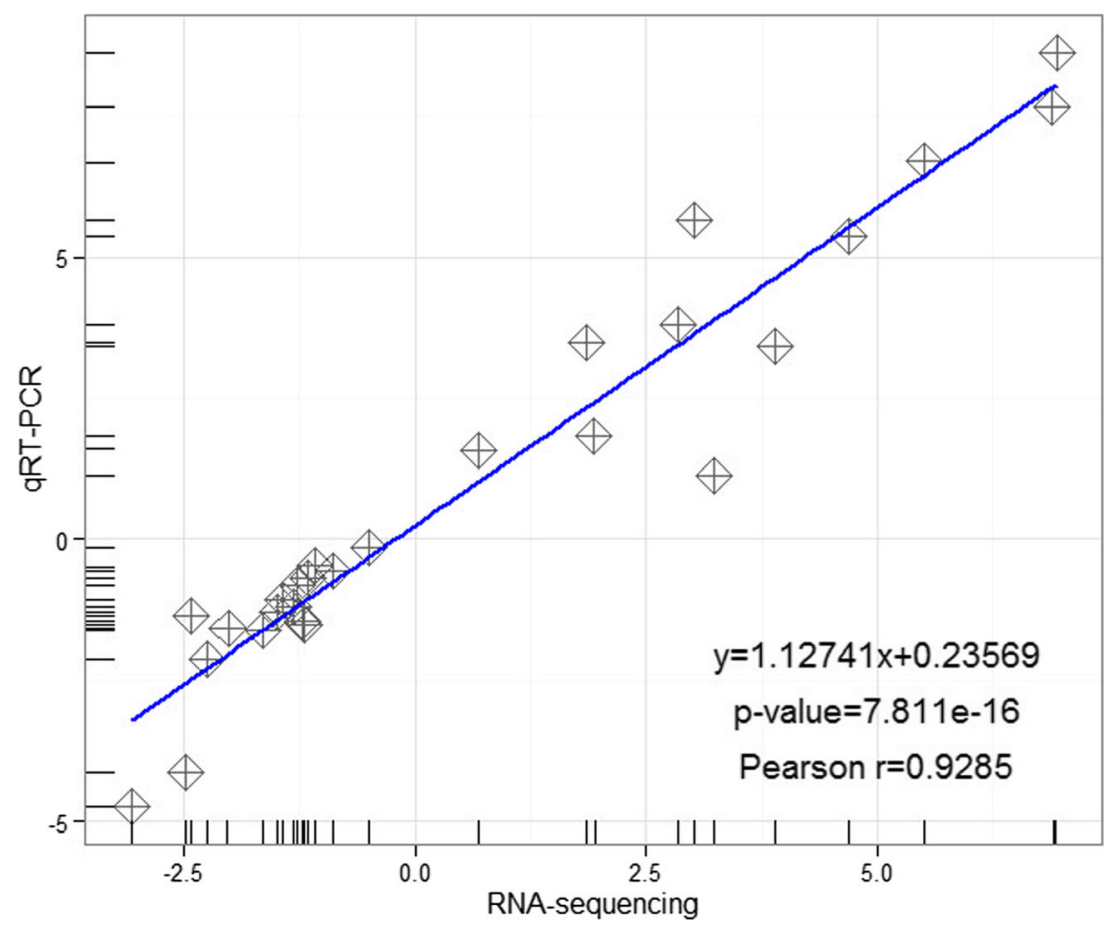

that $A B F$ played conserved roles in response to water-limited stress (Table 2). Ethylene signaling pathway has been well defined previously, and it is proven that ethylene interacts with ABA in response to stress (Stepanova and Alonso 2009). In ethylene signaling pathway, the CTR 1 (comp59632_c1_seq8), ETR (comp53115_c1_seq2), and EBF1/2 (comp54747_c0_seq2) homologues were identified in the dataset. Furthermore, $E B F 1 / 2$ was significantly up-regulated, indicating that ethylene signaling was an important process for $I$. sinensis to adapt to the changing environments. Thus, ABA and ethylene interactions form a complex network in response to the environmental changes (Cramer et al. 2011).

JA and SA signaling pathways are considered to play important roles in response to biotic stress (Li et al. 2014). COI1, JAZ, and JAR1 homologues were identified and uniformly up-regulated under TC. The results suggested that $I$. sinensis probably had additional $J A Z$ homologues, and they were negatively regulated by $C O I-1$ and $J A R 1$. In SA signaling pathway, pathogenesis-related $(P R)-1$ is required to induce SA signaling and bound by NPRI-TGA complex (Dong 2004). Two PR-1 homologues (comp44025_c1_seq1 and comp61194_c0_seq1) were identified and obviously downregulated with 23.80 and 15.99 -fold changes, respectively. The results indicated that SA signaling pathway played vital roles in response to stress, whereas more datasets should be supplied to expand global understandings for the SA regulations in Isoetes.

Carbohydrate metabolism is one of the most important primary processes and is associated with plants' survival
(McDowell 2011). Pathway enrichment analysis showed that 79 DEGs were significantly enriched in the "carbohydrate metabolism" category with 28 up-regulated and 51 downregulated genes (Table S6), further confirming pivotal roles in survival from basal species to higher plants. Starch and sucrose metabolisms have been well investigated recently, which are dynamic with the effective synthesis and degradation (Tang et al. 2014; Yang et al. 2003). Furthermore, starch and sucrose act as potential signals interacting with stress to adapt to the environmental changes (Yang et al. 2003). Important genes related to starch and sucrose metabolisms were present in this dataset and influenced by the environmental changes, indicating that $I$. sinensis shared a complex regulatory network for starch and sucrose metabolisms to adapt to the environmental changes (Bianchi et al. 1991; Tang et al. 2014). Moreover, the concentrations of starch and sucrose are often consistent with the related genes' regulations (McDowell 2011). Additional experiments need to demonstrate the consistency between genes' expressions and concentrations of starch and sucrose. Glycolysis is also involved in adaptation to environmental stress, such as drought, cold, and osmotic stresses (Plaxton 1996). Furthermore, a general upregulation of sucrose metabolism reveals enhanced glycolysis metabolisms in the level of transcription (Fernie et al. 2002; Fernie et al. 2004). In this study, DEGs related to glycolysis metabolisms were uniformly down-regulated, indicating that sucrose metabolism probably produced lots of energy under $\mathrm{SC}$ and it was mediated by glycolysis metabolism.

TFs are a group of proteins interacting with cis-regulatory elements of the target genes. An initial research states that TFs 
numbers are increasing from the algaes to dicots, such as 157 TFs in Cyanidioschyzon merolae, 1423 in Physcomitrella patens, and 2757 in Arabidopsis thaliana (Libault et al. 2009; Sharma et al. 2013). Furthermore, more complex organisms employ larger TFs to execute complicated regulations and metabolisms (Libault et al. 2009; Pérez-Rodríguez et al. 2009; Sharma et al. 2013). In this study, we identified 1646 putative TFs, further supporting the previous reports (Libault et al. 2009; Pigg 1992; Sharma et al. 2013). We further elucidated dynamic TF distributions with 134 up-regulated and 46 down-regulated TFs, which have been reported to play a pivotal role in response to the environmental changes (Hiz et al. 2014; Liu et al. 2014).

Multiple TF families are linked to abiotic or biotic stress, acting as an activator or repressor to regulate stress-induced changes, such as MYB, NAC, and WRKY proteins (Libault et al. 2009; Yu et al. 2012). Since the COLORED1 locus was identified to encode a MYB domain protein, a large number of MYB-related TFs have been accumulated and found to be involved in plant-specific processes, such as primary metabolism, cell fate, and response to biotic and abiotic stresses (Chen et al. 2013; Dubos et al. 2010). NAC proteins are plant-specific TFs, which play key roles in plant development and response to biotic or abiotic stress. A recent research has stated that 151 NAC genes in rice and 117 in Arabidopsis are identified using a genome-wide analysis (Olsen et al. 2005). Moreover, phylogenetic analyses reveal that NAC TFs are divided into NAC-A, NAC-B, and NAC-C subgroups (Nakashima et al. 2012). The NAC-A and NAC-B subgroups probably emerge after the separations between lycophytes and other vascular plants (Nakashima et al. 2012). There were substantial NAC sequences in the dataset and they were dynamic in response to the environmental changes. As a group of the basal vascular plants, the phylogenetic position and divergence time in Isoetes are particularly important, needing to do more investigations in the future.

bHLH proteins are a group of global TFs found in animals and plants. Although many members in this family have not been elucidated, a few $b H L H$ transcripts increase in drought, cold, and salt stresses, such as AtAIB and bHLH-92 (Jiang et al. 2009; Li et al. 2007). WRKY proteins in A. thaliana are known to encode novel CaM-binding TFs (Rushton et al. 2010). WRKY TFs not only act as activators but also as repressors to play key roles in depression and repression of plant important processes (Park et al. 2005). Multiple previous reports have demonstrated that WRKY TFs participate in various abiotic or biotic stress responses (Rushton et al. 2010). Furthermore, single WRKY protein often involves in several stresses to regulate the transcriptional reprograms (Rushton et al. 2010). Eight WRKY TFs were identified and uniformly up-regulated under TC, indicating that WRKY TFs played a vital role in response to the water-limited environments (Chen et al. 2012).

\section{Abbreviations}

BP, biological process; CC, cellular component; DEGs, differentially expressed genes; GO, gene ontology; JA, jasmonic acid; KEGG, Kyoto Encyclopedia of Genes and Genomes; MF, molecular function; NCBI, National Center of Biotechnology Information; Nr, non-redundant; N50, half of the assembled length; qRT-PCR, quantitative real-time PCR; SA, salicylic acid; SC, submerged condition; TC, terrestrial condition; $\mathrm{TF}$, transcription factor.

Acknowledgments The research was supported by the National Natural Science Foundation of China (30870168 and 31170203) and the Fundamental Research Funds for the Central Universities (2014204020206).

Open Access This article is distributed under the terms of the Creative Commons Attribution 4.0 International License (http:// creativecommons.org/licenses/by/4.0/), which permits unrestricted use, distribution, and reproduction in any medium, provided you give appropriate credit to the original author(s) and the source, provide a link to the Creative Commons license, and indicate if changes were made.

\section{References}

Andrews S (2010) FastQC: A quality control tool for high-throughput sequence data reference source

Baerenfaller K et al. (2012) Systems-based analysis of Arabidopsis leaf growth reveals adaptation to water deficit. Mol Syst Biol 8

Bianchi G, Gamba A, Murelli C, Salamini F, Bartels D (1991) Novel carbohydrate metabolism in the resurrection plant Craterostigma plantagineum. Plant J 1:355-359

Bustin SA et al (2009) The MIQE guidelines: minimum information for publication of quantitative real-time PCR experiments. Clin Chem 55:611-622

Chen L, Song Y, Li S, Zhang L, Zou C, Yu D (2012) The role of WRKY transcription factors in plant abiotic stresses. Biochim Biophys Acta BBA-Gene Regul Mech 1819:120-128

Chen Y, Chen Z, Kang J, Kang D, Gu H, Qin G (2013) AtMYB14 regulates cold tolerance in Arabidopsis. Plant Mol Biol Report 31: $87-97$

Chinnusamy V, Gong Z, Zhu JK (2008) Abscisic acid-mediated epigenetic processes in plant development and stress responses. J Integr Plant Biol 50:1187-1195

Cramer GR, Urano K, Delrot S, Pezzotti M, Shinozaki K (2011) Effects of abiotic stress on plants: a systems biology perspective. BMC Plant Biol 11:163

Der JP, Barker MS, Wickett NJ, Wolf PG (2011) De novo characterization of the gametophyte transcriptome in bracken fern, Pteridium aquilinum. BMC Genomics 12:99

Dong X (2004) NPR1, all things considered. Curr Opin Plant Biol 7:547552

Dubos C, Stracke R, Grotewold E, Weisshaar B, Martin C, Lepiniec L (2010) MYB transcription factors in Arabidopsis. Trends Plant Sci 15:573-581

Fernie A, Tiessen A, Stitt M, Willmitzer L, Geigenberger P (2002) Altered metabolic fluxes result from shifts in metabolite levels in sucrose phosphorylase-expressing potato tubers. Plant Cell Environ 25:1219-1232 
Fernie AR, Carrari F, Sweetlove LJ (2004) Respiratory metabolism: glycolysis, the TCA cycle and mitochondrial electron transport. Curr Opin Plant Biol 7:254-261

Foster AS, Gifford EM (1959) Comparative morphology of vascular plants

Gao J, Yu X, Ma F, Li J (2014) RNA-seq analysis of transcriptome and glucosinolate metabolism in seeds and sprouts of broccoli (Brassica oleracea var. italic). PLoS ONE 9:e88804

Grabherr MG et al (2011) Full-length transcriptome assembly from RNAseq data without a reference genome. Nat Biotechnol 29:644-652

Haas BJ et al (2013) De novo transcript sequence reconstruction from RNA-seq using the Trinity platform for reference generation and analysis. Nat Protoc 8:1494-1512

Hiz MC, Canher B, Niron H, Turet M (2014) Transcriptome analysis of salt tolerant common bean (Phaseolus vulgaris L.) under saline conditions. PLoS ONE 9:e92598

Hoot SB, Taylor WC (2001) The utility of nuclear ITS, a LEAFY homo$\log$ intron, and chloroplast atpB-rbcL spacer region data in phylogenetic analyses and species delimitation in Isoetes. Am Fern J 91: 166-177

Jiang Y, Yang B, Deyholos MK (2009) Functional characterization of the Arabidopsis bHLH92 transcription factor in abiotic stress. Mol Gen Genomics 282:503-516

Kanehisa M (2002) The KEGG database silico simulation of biological processes 247:91-103

Keeley J (1987) The adaptive radiation of photosynthetic modes in the genus Isoetes (Isoetaceae). Plant life in aquatic and amphibious habitats: $113-128$

Li H, Sun J, Xu Y, Jiang H, Wu X, Li C (2007) The bHLH-type transcription factor AtAIB positively regulates ABA response in Arabidopsis. Plant Mol Biol 65:655-665

Li Z, Han Q, Chen Y, Li W (2012) Microsatellite primers in the endangered quillwort Isoetes hypsophila (Isoetaceae) and crossamplification in I. sinensis. Am J Bot 99:e184-e186

$\mathrm{Li} \mathrm{H}$ et al (2014) RNA-seq derived identification of differential transcription in the chrysanthemum leaf following inoculation with Alternaria tenuissima. BMC Genomics 15:9

Libault M, Joshi T, Benedito VA, Xu D, Udvardi MK, Stacey G (2009) Legume transcription factor genes: what makes legumes so special? Plant Physiol 151:991-1001

Liu X, Wang Y, Wang Q, Guo Y (2001) Chromosome numbers of the Chinese Isoetes and their taxonomical significance. Acta Phytotax Sin 40:351-356

Liu X, Gituru WR, Wang QF (2004) Distribution of basic diploid and polyploid species of Isoetes in East Asia. J Biogeogr 31:1239-1250

Liu J-H, Peng T, Dai W (2014) Critical cis-acting elements and interacting transcription factors: key players associated with abiotic stress responses in plants. Plant Mol Biol Report 32:303-317

Livak KJ, Schmittgen TD (2001) Analysis of relative gene expression data using real-time quantitative PCR and the 2- $\Delta \Delta \mathrm{CT}$ method. Methods 25:402-408

McDowell NG (2011) Mechanisms linking drought, hydraulics, carbon metabolism, and vegetation mortality. Plant Physiol 155:1051-1059

Mortazavi A, Williams BA, McCue K, Schaeffer L, Wold B (2008) Mapping and quantifying mammalian transcriptomes by RNA-seq. Nat Methods 5:621-628

Nakashima K, Takasaki H, Mizoi J, Shinozaki K, Yamaguchi-Shinozaki $\mathrm{K}$ (2012) NAC transcription factors in plant abiotic stress responses. Biochim Biophys Acta (BBA) - Gene Regul Mech 1819:97-103

Olsen AN, Ernst HA, Leggio LL, Skriver K (2005) NAC transcription factors: structurally distinct, functionally diverse. Trends Plant Sci 10:79-87

Park CY et al (2005) WRKY group IId transcription factors interact with calmodulin. FEBS Lett 579:1545-1550
Peleg Z, Blumwald E (2011) Hormone balance and abiotic stress tolerance in crop plants. Curr Opin Plant Biol 14:290-295

Pérez-Rodríguez P, Riano-Pachon DM, Corrêa LGG, Rensing SA, Kersten B, Mueller-Roeber B (2009) PlnTFDB: updated content and new features of the plant transcription factor database. Nucleic acids research: gkp805

Pigg KB (1992) Evolution of isoetalean lycopsids. Ann Missouri Bot Gard:589-612

Pigg KB (2001) Isoetalean lycopsid evolution: from the Devonian to the present. Am Fern J 91:99-114

Plaxton WC (1996) The organization and regulation of plant glycolysis. Annu Rev Plant Biol 47:185-214

Riaño-Pachón DM, Ruzicic S, Dreyer I, Mueller-Roeber B (2007) PlnTFDB: an integrative plant transcription factor database. BMC Bioinforma 8:42

Rice P, Longden I, Bleasby A (2000) EMBOSS: the European molecular biology open software suite. Trends Genet 16:276-277

Rushton PJ, Somssich IE, Ringler P, Shen QJ (2010) WRKY transcription factors. Trends Plant Sci 15:247-258

Shan X, Li Y, Jiang Y, Jiang Z, Hao W, Yuan Y (2013) Transcriptome profile analysis of maize seedlings in response to high-salinity, drought and cold stresses by deep sequencing. Plant Mol Biol Report 31:1485-1491

Sharma N, Bhalla PL, Singh MB (2013) Transcriptome-wide profiling and expression analysis of transcription factor families in a liverwort, Marchantia polymorpha. BMC Genomics 14:915

Shih M-C et al (2014) BeMADS1 is a key to delivery MADSs into nucleus in reproductive tissues - de novo characterization of Bambusa edulis transcriptome and study of MADS genes in bamboo floral development. BMC Plant Biol 14:179

Stepanova AN, Alonso JM (2009) Ethylene signaling and response: where different regulatory modules meet. Curr Opin Plant Biol 12: $548-555$

Tang S et al. (2014) Analysis of the drought stress-responsive transcriptome of black cottonwood (Populus trichocarpa) using deep RNA sequencing. Plant Mol Biol Report:1-15

Taylor WC, Hickey RJ (1992) Habitat, evolution, and speciation in Isoetes. Ann Missouri Bot Gard:613-622

Wang W, Vinocur B, Altman A (2003) Plant responses to drought, salinity and extreme temperatures: towards genetic engineering for stress tolerance. Planta 218:1-14

Wang JY, Gituru RW, Wang QF (2006) Ecology and conservation of the endangered quillwort Isoetes sinensis in China. J Nat Hist 39:40694079

Yamaguchi-Shinozaki K, Shinozaki K (2006) Transcriptional regulatory networks in cellular responses and tolerance to dehydration and cold stresses. Annu Rev Plant Biol 57:781-803

Yang J, Zhang J, Wang Z, Zhu Q, Liu L (2003) Activities of enzymes involved in sucrose-to-starch metabolism in rice grains subjected to water stress during filling. Field Crop Res 81:69-81

Ye J et al (2006) WEGO: a web tool for plotting GO annotations. Nucleic Acids Res 34:W293-W297

Yoshida T et al (2010) AREB1, AREB2, and ABF3 are master transcription factors that cooperatively regulate ABRE-dependent ABA signaling involved in drought stress tolerance and require $\mathrm{ABA}$ for full activation. Plant J 61:672-685

Yu S, Zhang F, Yu Y, Zhang D, Zhao X, Wang W (2012) Transcriptome profiling of dehydration stress in the Chinese cabbage (Brassica rapa L. ssp. pekinensis) by tag sequencing. Plant Mol Biol Report 30:17-28

Zeng $C$ et al (2014) Chilling acclimation provides immunity to stress by altering regulatory networks and inducing genes with protective functions in cassava. BMC Plant Biol 14:207 